\title{
Design and Testing of the AGS Booster BPM Detector ${ }^{*}$ BNL--45411
}

\author{
R. Thomas, D. J. Ciardullo, and W. Van Zwienen \\ AGS Deparment \\ Brookhaven National Laboratory \\ Upton, New York 11973-5000
}

DE91 013332

\section{Abstract}

The AGS Booster beam position monitor system must accurately measure the position of beams and bunches over a wide range of intensity. The frequency of operation must also cover a wide range $(600 \mathrm{kHz}$ to $4.2 \mathrm{MHz})$ since the Booster accelerates both protons and heavy ions. Split-cylinder electrodes were chosen to monitor the position of the beam because of their good low frequency response and high linearity. The need to accelerate low-energy partially-stripped heavy ions requires the pick-up electrodes (PUEs) to operate in a $3 \times 10^{-11}$ tor vacuum. The PUEs are to measure the beam position to an absolute accuracy of $\pm 0.5 \mathrm{~mm}$ and must therefore be mechanically stable despite the requirement that they be vacuum fired at $950^{\circ} \mathrm{C}$ and baked periodically to $300^{\circ} \mathrm{C}$. This presentation describes both the mechanical design of the PUEs and the automated test procedure used to ensure the stability, accuracy, and linearity of each unit.

\section{DESIGN}

Each capacitive detector is made from a diagonally split cylinder (Fig. 1). The position monitor also incorporates a calibration ring which couples equally w both electrodes. The ring provides a simulation of the electrical zero position that allows the monitor to be checked [1]. (See Fig. 1.)

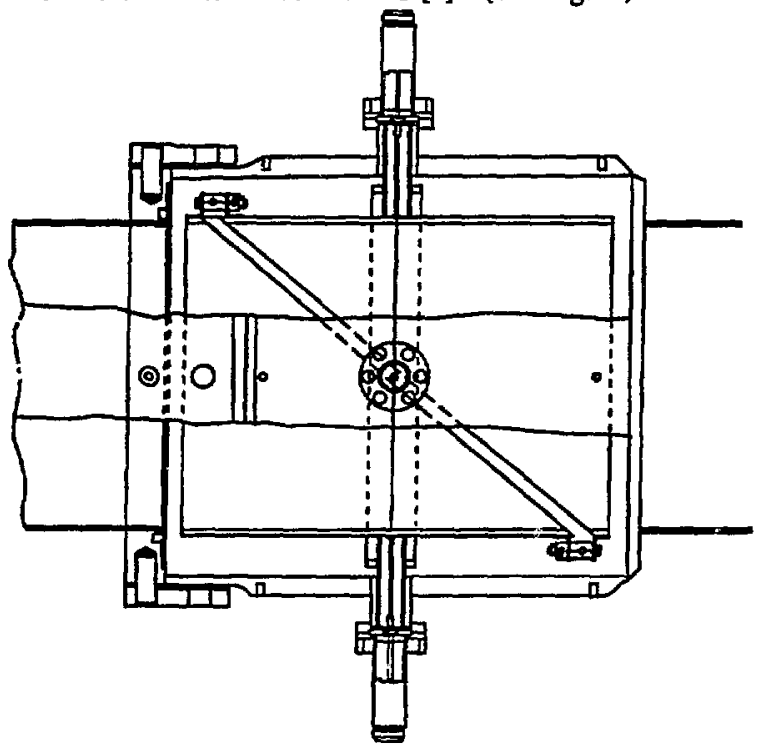

Figure 1. Beam position monitor (longiudinal view).

Work supported by the U. S. Department of Energy,
The completely assembled pusition monitor must be vacuum-fired, i.e., heated in a vacuum, at $950^{\circ} \mathrm{C}$ to remove dissolved hydrogen from the stainiess steel components in order to achieve the desired $10^{-11}$ torr vacuum. Therefore a special design was required to suppor the components that permitted thermal expansion to occur without creating forces which could ca'rse deformation or fracture. Stainless steel grows linearly by absut $0.018 \mathrm{~mm} / \mathrm{mm}$ when heated from room temperature to $950^{\circ} \mathrm{C}$. Hence, a stainless cylinder $127 \mathrm{~mm}$ in diameter and $127 \mathrm{~mm}$ long will grow approximately $2.3 \mathrm{~mm}$ in dianeter and length. Spring material could not be used because at $950^{\circ} \mathrm{C}$, the stiffiness coefficient of even high temperature spring material decreases by $90 \%$.

In addition to accommodating the large thermal expansion, the various components must be electrically isolated from one another. The dielectric material needs to have the following properties: very low outgassing and very low porosity, ability to withstand vacuum firing to $950^{\circ} \mathrm{C}$, and good structural strength. High-density alumina, a ceramic containing more than $92 \% \mathrm{Al}_{2} \mathrm{O}_{3}$, is suitable. The thermal growth of alumina, however, is about half that of stainless steel over the same ternperature range, so in the design, the stainless always surrounds the alumina so that the stainless grows away from the alumina as the temperature increases.

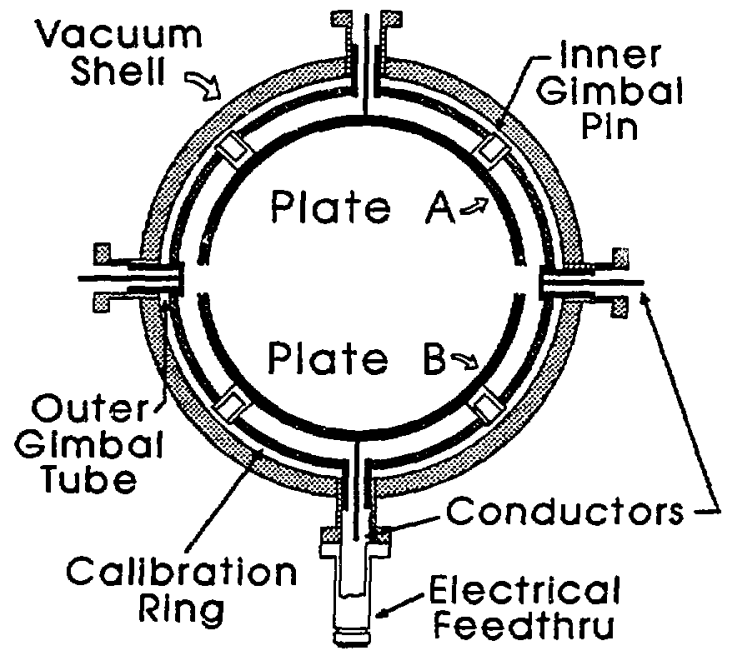

Figure 2. Double-gimballed beam position monitor.

Fig. 2 shows the arrangement of the four outer ceramic tubes which provide the gimbal support of the calibration ring from the outer shell. The four inner ceramic pins support the split-cylinder assembly from the calibration ring and thereby from the outer shell as weil. Because all the support members are located in a plane perpendicular to the longitudinal (beam) centerline, expansion and contraction of the components can 
occur freely in the longitudinal direction without inducing any loading on other components or on the ceramic supports. Radial movement is accommodated by the gimbal support between the outer shell and the calibration ring and between the catibration ring and the split-cylinder assembly. Differential radial expansion berween any of the three cylindrical components, outer shell, calibration ring, or split-cylinder assembly, is accommodated by a radial sliding motion between either of the metal components and the ceramic supports without imposing loads on any of the components.

In addition to accommodating thermal movement, the design maintains the alignment of the components, including concentricity. The gimballed support insures that concentricity will be maintained even when thermal gradients produce relative dimensional changes.

To obtain the required accuracy of the location of the components, diametral clearances between the ceramic pins and tubes and their corresponding holes were limited to $<0.03 \mathrm{~mm}$. In order to permit assembly of the gimbals, the holes themselves had to be located with an similar accuracy. It was also necessary to fully anneal the stainless stcel components before machining, and in some cases to stress relieve them prior to finish machining, in order to minimize distortion resulting from machining stresses or from the $950^{\circ} \mathrm{C}$ vacuum firing. Any distortion during firing can cause binding at the gimbal connections which, in tum, imposes loads on the adjacent stainless and ceramic components that can result in damage to, or failure of, one or more components.

To maintain the required gap betwcen the halves of the diagonally-cut cylinder while holding them sogether as a unit for gimbal mounting, it was necessary to fasten them together semi-rigidly while maintaining electrical isolation between the plates as shown in the Fig. 3. Two ceramic pins span the gap at opposite ends. Although litile differential thermal growth

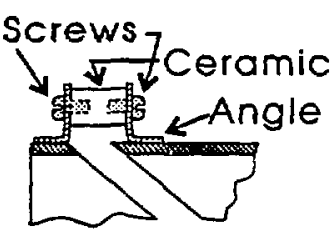

Plates was anticipated, the stainless steel angles to which the ceramic pins are attached flex to allow for any small changes in the gap during heating.

The plates of the detector are fabricated from a cylinder which was machined from fully annealed stainless tube stock

Figure 3. Split-cylinder assemThe cylinder was then stress bly. relieved and the gimbal mounting holes added. The two pairs of holes are offset, so that once the balves are separated by the desired gap, the holes are co-planar. Even though it is a relatively slow process, electric discharge cutting was used so that no machining stresses were introduced into the part.

\section{TEST}

\section{A. Description}

After the split-cylinder pick-up electrodes and the associated calibration ring have been carefully assembled, the unit is inserted into the pick-up electrode body or shell. The pick-up electrode body is itself part of the vacuum chamber. It has openings for the four coaxial signal feed-throughs as well as mounting holes to allow the BPM to be precisely positioned in the Booster quadrupoles.
In order to allow the split cylinder to be inserted into the outer shell, one end is left open until it is welded to the flange that reduces the diameter of the chamber to that which passes through the quadrupoles. As a consequence, one of the halves of the split cylinder does not see the electrical environment it sees after being installed. Therefore, it is necessary to attempt to produce an electrically similar configuration. This is done by using a short extension.

After the extension has been attached, the detector is positioned vertically on a stand. Short rods are inserted into the same mounting holes which allow the BPM to be precisely mounted in the quadrupoles. These rods accurately position the detector on the mounting stand and prevent it from moving.

In order to obtain the scan measurement data, a signal wire is dropped through the inside of the pick-up electrode body. The wire (a $0.25-\mathrm{mm}$ diameter piano wire) is connected to a $180 \Omega$ terminating resistor and is then placed under tension produced by a strong spring. The signal wire is strung between the two ams of a large $C$-shaped suppor. The C-shaped support itself is mounted to the platform of an X/Y Cross Table (Techno Model HL32SBM2298).

A program running on a Hewlett Packard 9836 S workstation/instmument controller automates the measurement process. The primary measuring instrument is the HP3577A Network Analyzer. The RF outut port of the HP3577A provides the signal, and the $A$ and $B$ receiver ports measure the r.m.s. voltage on the each half of the split-cylinder of the pick-up electrode assembly. The equipment used is schematically indicated in Figure 4.

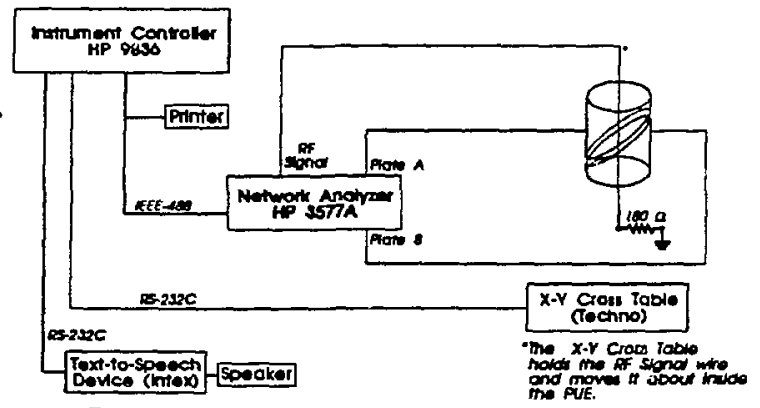

Figure 4. Set-up for the scamed wire measurements.

The X/Y Table Controller is attached to one of two RS$232 \mathrm{C}$ interfaces on the workstation. A complete scan consists of 261 measurements. By convention, the plane of highest sensitivity is designated $y$. The aperture is scanned at five $x$ positions $(-50,-30,0,+30,+50)$. At each of the $x$ positions, a measurement is taken every $2 \mathrm{~mm}$ from $y=-50 \mathrm{~mm}$ to $y=+50$ $\mathrm{mm}$ (51 measurements). In addition to these 255 measurements, the wire is moved to $(0,0)$ between each $x$ position scan and at the beginning and the end of the entire measurement process, thus giving 6 additional measurements at position $(0,0)$.

For each of the 261 measurements, the Network Analyzer takes 401 measurements each of the r.m.s. magnitude of the voltage of the signals at its $A$ and $B$ receivers. (These 401 measurements are called a "trace".) The program retrieves these values and computes the average, the difference between the maximum and minimum measurement, and the standard deviation for the two signals. These three values for each 
channel are stored on flexible discs along with the coordinates of the wire position.

\section{B. X/Y Table Accuracy}

The X/Y Cross Arm Table is an economical model and can not provide a continuous indication of its position. In fact, the table controller only knows when the platform has reached a single location on each of the axes, and this single focation must be at one of the extremes of the arm movement. The controller knows that this position has been reached by the opening of a micro-switch when a protrusion mounted to the platform contacts it. All positions are then relative to the position of the platform when the switches on each axis opens (the "Home" position). The controller sends pirises to the two stepper motors to drive a lead screw which in tum moves the platform relative to the Home position. There are 100 halfsteps per mm.

When the table is commanded to Home, the platform moves at the programmed acceleration until the switches are struck. The manufacturer specifies the accuracy of the homing operation to be $\pm 0.01 \mathrm{~mm}$. In addition to this error must be added the inaccuracy of determining the position of the mechanical center of the detector as mounted on the stand relative to the Home position.

The center of the stand (relative to the Home position) is determined by use of a special centering fixture. The centering fixture is mounted on the stand in place of the detector. The signal wire is dropped through a small hole in the fixture and made taut Two micrometers are mounted over the center hole on a vertical bar. The deviation of the center of the wire-the wire has a radius of about 5 mils-from the true center is determined using the micrometers. The vertical mounting of two micrometers allows the C-shaped suppor to be adjusted to make the wire perpendictlar to the stand. After the wire has been centered along one axis and ruade perpendicular in that plane, the centering fixture is rotated $90^{\circ}$ and the procedure is repeated for the other axis. When the centering was repeatable to $\pm 0.08 \mathrm{~mm}$, it was deemed acceptable.

In addition to the difficulty of centering the wire, there was initially some jitter in the movement of the table platform itself. It was determined that this was due to either a bowing of the lead screw or to a loose fit that allowed the platform to tilt first to one side and then the other as it progressed along the lead screw. The effect was seen in the plots of the deviation of the data from a straight line where it manifested itself as a periodic variation instead of a smooth curve. This problem was solved by dismanting the entire $X / Y$ Table and reassembling it with atuention being given to making everything tight.

\section{Results}

Immediately after the data have been taken and stored, it is analyzed. All the results are ploued and a robust straight line fit is made to each of the five data sets (one for each of the five $x$ positions $)$ for the points which falls within $-30 \mathrm{~mm}$ $<y<+30 \mathrm{~mm}$ [2]. The fits are two-parameter fits (slope and offset) of $\left(V_{A}-V_{B}\right) /\left(V_{A}+V_{B}\right)$ as a function of the programmed $y$ position, where $V_{A}$ and $V_{B}$ are the magnitudes of the voltages on plates $A$ and $B$ of the PUE, respectively. A final fit is performed for the same range of $y$ values, but combining all the points in this range for the three central $x$ scans $(-30,0,+30)$.

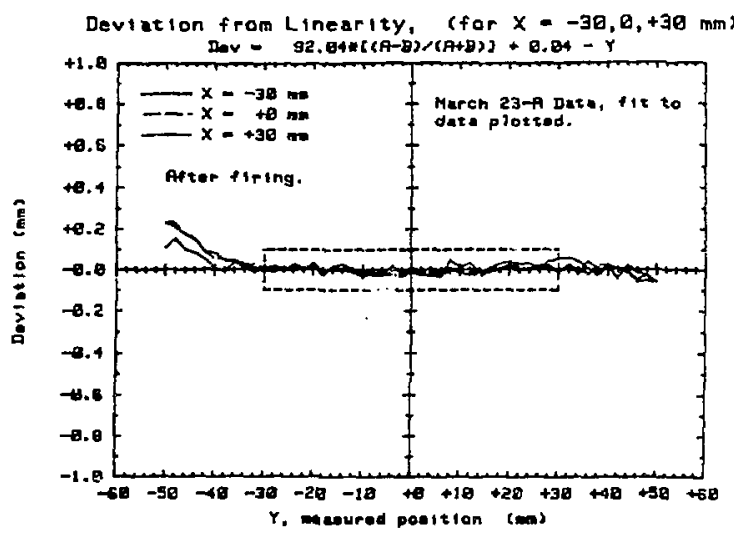

Figure 5. Typical scan wire measurement results.

The deviations from a straight line are plotted in all cases. Typical results are shown in Fig. 5.

Sixty detectors have been measured. (Forty-six are required for the Booster and six more are needed for the transfer line from the Booster to the AGS.) The average value for the slope, $K$, was 90.75 at $5 \mathrm{MHz}$. The standand deviation was 0.20 , but the $K$ value for eacn individual detector will be used by the Booster Beam Position Monitoring System. The offset, 0 , averaged $-0.26 \mathrm{~mm}$. All but three detectors had negative offsets. This is believed to result from the inability of the extender to fully simulate the electrical environment of the detector as it is after the shell has been welded to the next section of the vacuum chamber. Therefore, the offsets that are automatically acquired during operation by the Booster control software will be used instead [3].

\section{ACKNOWLEDGMENTS}

The authors wish to thank August Hoffmann, who carefully assembled and tested the position monitors, for his helpful suggestions, and James Savino for his pyork on the stand for the X/Y Cross Table.

\section{REFERENCES}

[1] E. Beadle, et. al., "Design of the AGS Booster Beam Position Monitor System," in Proceedings of the 1989 IEEE Particle Accelerator Conference, Vol. 3, Chicago, IL, Maxch 1989, pp. 1536-1538.

[2] W. H. Press, et. al . Numerical Recipes, Cambridge: Cambridge University Press, 1986, pp. 539-546.

[3] D. J. Cisrdullo, et. al." "The AGS Booster Beam Position Monicor System," in Proceedings of the 1991 IEEE Particle Accelerator Conference, San Francisco, CA. May 1991, to be published. 